\title{
Best approximation of a nonlinear fractional Volterra integro-differential equation in matrix MB-space
}

\author{
Reza Chaharpashlou ${ }^{1 *}$ and Reza Saadati ${ }^{*}$
}

\section{"Correspondence:}

r_chaharpashlou@mathdep.iust.ac.ir; rsaadati@eml.cc; rsaadati@iust.ac.ir

${ }^{1}$ School of Mathematics, Iran

University of Science and

Technology, Narmak, Tehran, Iran

\begin{abstract}
In this article, we introduce a class of stochastic matrix control functions to stabilize a nonlinear fractional Volterra integro-differential equation with $\Psi$-Hilfer fractional derivative. Next, using the fixed-point method, we study the Ulam-Hyers and Ulam-Hyers-Rassias stability of the nonlinear fractional Volterra integro-differential equation in matrix MB-space.
\end{abstract}

MSC: 54C40; 14E20; 46E25; 47H10

Keywords: Hyers-Ulam stability; Fractional Volterra integral; $\Psi$-Hilfer fractional derivative; Integro-differential equations; Matrix MB-space

\section{Introduction}

Fractional calculus is considered as a branch of mathematical analysis which deals with the investigation and applications of integrals and derivatives of arbitrary order. Therefore, fractional calculus is an extension of the integer-order calculus that considers integrals and derivatives of any real or complex order $[1,2]$, i.e., unifies and generalizes the notions of integer-order differentiation and $n$-fold integration.

Different forms of fractional operators have been introduced, like the RiemannLiouville, Grinwald-Letnikov, Weyl, Caputo, Marchaud, and Hadamard fractional derivatives. The first approach is that Riemann-Liouville, which is based on iterating the classical integral operator $n$ times and then considering the Cauchy's formula where $n$ ! is replaced by the Gamma function, and hence the fractional integral of noninteger order is defined.

Fractional calculus has attracted the attention of many mathematicians, but also of some researchers in other areas like physics, chemistry, and engineering. As it is well known, several physical phenomena are often better described by fractional derivatives. This is mainly due to the fact that fractional operators take into consideration the evolution of the system, by taking the global correlation, and not only local characteristics. Moreover, integer-order calculus sometimes contradicts the experimental results, and therefore, derivatives of fractional order may be more suitable [3-5].

Very useful physical applications have given birth to the variable-order fractional calculus, for example, in modeling mechanical behaviors [6]. Nowadays, variable-order frac-

(c) The Author(s) 2021. This article is licensed under a Creative Commons Attribution 4.0 International License, which permits use, sharing, adaptation, distribution and reproduction in any medium or format, as long as you give appropriate credit to the original author(s) and the source, provide a link to the Creative Commons licence, and indicate if changes were made. The images or other third party material in this article are included in the article's Creative Commons licence, unless indicated otherwise in a credit line to the material. If material is not included in the article's Creative Commons licence and your intended use is not permitted by statutory regulation or exceeds the permitted use, you will need to obtain permission directly from the copyright holder. To view a copy of this licence, visit http://creativecommons.org/licenses/by/4.0/. 
tional calculus is particularly recognized as a useful and promising approach in the modeling of diffusion processes, in order to characterize time- or concentration-dependent anomalous diffusion, or diffusion processes in inhomogeneous porous media [7].

Results on existence and stability of solutions of implicit fractional differential equations can be found in [8-11].

By proposing the study of solution stability via fractional integrals and fractional derivatives, we can generalize the results and obtain the usual ones as particular cases. In this article, we study distribution functions with the ranges in a class of matrix algebras with the generalized triangular norms, to define MB-space and introduce a new class of matrix control functions. Also, we will use two recent fractional operators, that is, of general differentiation and integration [12].

These concepts help us study the Hyers-Ulam (in short HU) and Hyers-Ulam-Rassias (in short HUR) stability of fractional nonlinear Volterra integro-differential equation (in short VIDE),

$$
\left\{\begin{array}{l}
H_{\mathbb{D}_{0+}^{l, \kappa ; \Psi}} \mu(\varsigma)=\boldsymbol{F}(\varsigma, \mu(\varsigma))+\int_{0}^{\varsigma} \boldsymbol{H}(\varsigma, \vartheta, \mu(\varsigma)) d \vartheta \\
\mathcal{I}_{0+}^{1-\gamma} \mu(0)=\sigma
\end{array}\right.
$$

with $\varsigma \in[0, T]$ and a continuous function (in short CF) $\boldsymbol{F}(\varsigma, \mu)$, also $\boldsymbol{H}(\varsigma, \vartheta, \mu)$ is a CF with respect to $\zeta, \vartheta$ and $\mu$ on $[0, T] \times \mathbb{R} \times \mathbb{R}, \sigma$ is a fixed number, ${ }^{H} \mathbb{D}_{0+}^{\iota, \kappa ; \psi} \mu(\cdot)$ is defined in (2.1) in which $0<\iota<1,0 \leq \kappa \leq 1$, and $\mathcal{I}_{0+}^{1-\gamma}(\cdot)$ is the $\Psi$-Riemann-Liouville fractional integral in which $0 \leq \gamma<1[12]$.

\section{Preliminaries}

Here, we let $\Xi_{1}=[0, T]$, with $T>0, \Xi_{2}=(0, \infty), \Xi_{3}=(0,1], \Xi_{4}=[0, \infty]$, and $\Xi_{5}=[0,1]$ (note that $\Xi_{5}^{\circ}=(0,1)$ denotes the interior of $\Xi_{5}$ ).

Let

$$
\operatorname{diag} M_{n}\left(\Xi_{5}\right)=\left\{\left[\begin{array}{lll}
a_{1} & & \\
& \ddots & \\
& & a_{n}
\end{array}\right]=\operatorname{diag}\left[a_{1}, \ldots, a_{n}\right], a_{1}, \ldots, a_{n} \in \Xi_{5}\right\},
$$

where $\operatorname{diag} M_{n}\left(\Xi_{5}\right)$ is equipped with the partial order relation:

$$
\begin{array}{ll}
\boldsymbol{a}:=\operatorname{diag}\left[a_{1}, \ldots, a_{n}\right], \quad \boldsymbol{b}:=\operatorname{diag}\left[b_{1}, \ldots, b_{n}\right] \in \operatorname{diag} M_{n}\left(\Xi_{5}\right), \\
\boldsymbol{a} \preceq \boldsymbol{b} \Longleftrightarrow a_{j} \leq b_{j} \quad \text { for every } j=1, \ldots, n .
\end{array}
$$

Also, $\boldsymbol{a} \prec \boldsymbol{b}$ denotes that $\boldsymbol{a} \preceq \boldsymbol{b}$ and $\boldsymbol{a} \neq \boldsymbol{b} ; \boldsymbol{a} \ll \boldsymbol{b}$ and $a_{j}<b_{j}$ for every $j=1, \ldots, n$. We define $\boldsymbol{e}:=\operatorname{diag}[e, \ldots, e]$ in $\operatorname{diag} M_{n}\left(\boldsymbol{\Xi}_{5}\right)$ where $e \in \boldsymbol{\Xi}_{5}$. For example, $\mathbf{1}=\operatorname{diag}[1, \ldots, 1]$ and $\mathbf{0}=\operatorname{diag}[0, \ldots, 0]$.

Now, we extend the concept of triangular norms $[13,14]$ on $\operatorname{diag} M_{n}\left(\Xi_{5}\right)$.

Definition 2.1 A generalized triangular norm (in short GTN) on $\operatorname{diag} M_{n}\left(\Xi_{5}\right)$ is an operation $\circledast: \operatorname{diag} M_{n}\left(\Xi_{5}\right) \times \operatorname{diag} M_{n}\left(\Xi_{5}\right) \rightarrow \operatorname{diag} M_{n}\left(\Xi_{5}\right)$ satisfying the following conditions:

(a) $\left.\left(\forall \boldsymbol{a} \in \operatorname{diag} M_{n}\left(\Xi_{5}\right)\right)(\boldsymbol{a} \circledast \mathbf{1})=\boldsymbol{a}\right)$ (boundary condition);

(b) $\left(\forall(\boldsymbol{a}, \boldsymbol{b}) \in\left(\operatorname{diag} M_{n}\left(\Xi_{5}\right)\right)^{2}\right)(\boldsymbol{a} \circledast \boldsymbol{b}=\boldsymbol{b} \circledast \boldsymbol{a})$ (commutativity); 
(c) $\left(\forall(\boldsymbol{a}, \boldsymbol{b}, \boldsymbol{c}) \in\left(\operatorname{diag} M_{n}\left(\Xi_{5}\right)^{3}\right)(\boldsymbol{a} \circledast(\boldsymbol{b} \circledast \boldsymbol{c})=(\boldsymbol{a} \circledast \boldsymbol{b}) \circledast \boldsymbol{c})\right.$ (associativity);

(d) $\left(\forall\left(\boldsymbol{a}, \boldsymbol{a}^{\prime}, \boldsymbol{b}, \boldsymbol{b}^{\prime}\right) \in\left(\operatorname{diag} M_{n}\left(\Xi_{5}^{4}\right)\left(\boldsymbol{a} \preceq \boldsymbol{a}^{\prime}\right.\right.\right.$ and $\boldsymbol{b} \preceq \boldsymbol{b}^{\prime} \Longrightarrow \boldsymbol{a} \circledast \boldsymbol{b} \preceq \boldsymbol{a}^{\prime} \circledast \boldsymbol{b}^{\prime}$ (monotonicity).

For every $\boldsymbol{a}, \boldsymbol{b} \in \operatorname{diag} M_{n}\left(\Xi_{5}\right)$ and all sequences $\left\{\boldsymbol{a}_{k}\right\}$ and $\left\{\boldsymbol{b}_{k}\right\}$ converging to $\boldsymbol{a}$ and $\boldsymbol{b}$, respectively, suppose we have

$$
\lim _{k}\left(\boldsymbol{a}_{k} \circledast \boldsymbol{b}_{k}\right)=\boldsymbol{a} \circledast \boldsymbol{b},
$$

then, $\circledast$ on $\operatorname{diag} M_{n}\left(\Xi_{5}\right)$ is continuous GTN (in short CGTN). Now we present some examples of CGTN:

(1) If $\circledast_{M}: \operatorname{diag} M_{n}\left(\Xi_{5}\right) \times \operatorname{diag} M_{n}\left(\Xi_{5}\right) \rightarrow \operatorname{diag} M_{n}\left(\Xi_{5}\right)$ is defined by

$$
\boldsymbol{a} \circledast_{M} \boldsymbol{b}=\operatorname{diag}\left[t_{1}, \ldots, t_{n}\right] \circledast_{M} \operatorname{diag}\left[s_{1}, \ldots, s_{n}\right]=\operatorname{diag}\left[\min \left\{t_{1}, s_{1}\right\}, \ldots, \min \left\{t_{n}, s_{n}\right\}\right],
$$

then $\circledast_{M}$ is CGTN (minimum CGTN);

(2) If $\circledast_{P}: \operatorname{diag} M_{n}\left(\Xi_{5}\right) \times \operatorname{diag} M_{n}\left(\Xi_{5}\right) \rightarrow \operatorname{diag} M_{n}\left(\Xi_{5}\right)$ is such that

$$
\boldsymbol{a} \circledast_{P} \boldsymbol{b}=\operatorname{diag}\left[t_{1}, \ldots, t_{n}\right] \circledast_{P} \operatorname{diag}\left[s_{1}, \ldots, s_{n}\right]=\operatorname{diag}\left[t_{1} \cdot s_{1}, \ldots, t_{n} \cdot s_{n}\right],
$$

then $\circledast_{P}$ is CGTN (product CGTN);

(3) If $\circledast_{L}: \operatorname{diag} M_{n}\left(\Xi_{5}\right) \times \operatorname{diag} M_{n}\left(\Xi_{5}\right) \rightarrow \operatorname{diag} M_{n}\left(\Xi_{5}\right)$ is defined by

$$
\begin{aligned}
\boldsymbol{a} \circledast_{L} \boldsymbol{b} & =\operatorname{diag}\left[t_{1}, \ldots, t_{n}\right] \circledast_{L} \operatorname{diag}\left[s_{1}, \ldots, s_{n}\right] \\
& =\operatorname{diag}\left[\max \left\{t_{1}+s_{1}-1,0\right\}, \ldots, \max \left\{t_{n}+s_{n}-1,0\right\}\right],
\end{aligned}
$$

then $\circledast_{P}$ is CGTN (Lukasiewicz CGTN).

Now, we present some numerical examples:

$$
\begin{aligned}
& \operatorname{diag}\left[\frac{3}{7}, 1, \frac{4}{5}\right] \circledast_{M} \operatorname{diag}\left[0, \frac{1}{5}, \frac{2}{3}\right]=\left[\begin{array}{lll}
\frac{3}{7} & & \\
& 1 & \\
& & \frac{4}{5}
\end{array}\right] \circledast_{M}\left[\begin{array}{lll}
0 & & \\
& \frac{1}{5} & \\
& & \frac{2}{3}
\end{array}\right]=\left[\begin{array}{llll}
0 & & \\
& \frac{1}{5} & \\
& & \frac{2}{3}
\end{array}\right] \\
& =\operatorname{diag}\left[0, \frac{1}{5}, \frac{2}{3}\right] \text {, } \\
& \operatorname{diag}\left[\frac{3}{7}, 1, \frac{4}{5}\right] \circledast_{P} \operatorname{diag}\left[0, \frac{1}{5}, \frac{2}{3}\right]=\left[\begin{array}{lll}
\frac{3}{7} & & \\
& 1 & \\
& & \frac{4}{5}
\end{array}\right] \circledast_{P}\left[\begin{array}{lll}
0 & & \\
& \frac{1}{5} & \\
& & \frac{2}{3}
\end{array}\right]=\left[\begin{array}{llll}
0 & & \\
& \frac{1}{5} & \\
& & \frac{8}{15}
\end{array}\right] \\
& =\operatorname{diag}\left[0, \frac{1}{5}, \frac{8}{15}\right] \text {, } \\
& \operatorname{diag}\left[\frac{3}{7}, 1, \frac{4}{5}\right] \circledast_{L} \operatorname{diag}\left[0, \frac{1}{5}, \frac{2}{3}\right]=\left[\begin{array}{lll}
\frac{3}{7} & & \\
& 1 & \\
& & \frac{4}{5}
\end{array}\right] \circledast_{L}\left[\begin{array}{lll}
0 & & \\
& \frac{1}{5} & \\
& & \frac{2}{3}
\end{array}\right]=\left[\begin{array}{llll}
0 & & \\
& \frac{1}{5} & \\
& & \frac{7}{15}
\end{array}\right] \\
& =\operatorname{diag}\left[0, \frac{1}{5}, \frac{7}{15}\right] \text {. }
\end{aligned}
$$


Then we get

$$
\begin{aligned}
& \operatorname{diag}\left[\frac{3}{7}, 1, \frac{4}{5}\right] \circledast_{M} \operatorname{diag}\left[0, \frac{1}{5}, \frac{2}{3}\right] \\
& \succeq \operatorname{diag}\left[\frac{3}{7}, 1, \frac{4}{5}\right] \circledast_{P} \operatorname{diag}\left[0, \frac{1}{5}, \frac{2}{3}\right] \\
& \geq \operatorname{diag}\left[\frac{3}{7}, 1, \frac{4}{5}\right] \circledast_{L} \operatorname{diag}\left[0, \frac{1}{5}, \frac{2}{3}\right] .
\end{aligned}
$$

We consider the set $D^{+}$of matrix-distribution-function-valued (MDF-valued), left continuous and increasing functions $\varphi: \mathbb{R} \cup\{-\infty, \infty\} \rightarrow \operatorname{diag} M_{n}\left(\Xi_{5}\right)$ such that $\varphi_{0}=\mathbf{0}$ and $\varphi_{+\infty}=\mathbf{1}$. Now $O^{+} \subseteq D^{+}$are all (proper) functions $\varphi \in D^{+}$for which $\ell^{-} \varphi_{+\infty}=\mathbf{1}\left(\ell^{-} \varphi_{\tau}=\right.$ $\left.\lim _{\zeta \rightarrow \tau^{-}} \varphi_{\zeta}\right)$. Note that proper MDF-valued functions are the MDF-valued functions of real random variables (i.e., of those random variables $g$ that a.s. take real values $(P(|g|=$ $\infty)=0)$ ).

In $D^{+}$, we define " $\preceq$ " as follows:

$$
\varphi \precsim \phi \quad \Longleftrightarrow \varphi_{\tau} \preceq \phi_{\tau}, \quad \forall \tau \in \mathbb{R} .
$$

Also for each $\varsigma \in \mathbb{R}$,

$$
\nabla_{\tau}^{\varsigma}= \begin{cases}\mathbf{0}, & \text { if } \tau \leq \varsigma \\ \mathbf{1}, & \text { if } \tau>\varsigma\end{cases}
$$

belongs to $D^{+}$and for every MDF-valued $\varphi$ we have $\varphi \precsim \nabla^{0}[13,15]$. For example,

$$
\varphi_{\tau}= \begin{cases}\mathbf{0}, & \text { if } \tau \leq 0, \\ \operatorname{diag}\left[1-e^{-\tau}, \frac{\tau}{1+\tau}, e^{-\frac{1}{\tau}}\right], & \text { if } \tau>0\end{cases}
$$

is an MDF-valued function in $\operatorname{diag} M_{3}\left(\Xi_{5}\right)$. Note that $\varphi_{\tau}=\operatorname{diag}\left[\varphi_{1, \tau}, \ldots, \varphi_{n, \tau}\right]$, with $\varphi_{i, \tau}$ being distribution functions, is MDF-valued.

Definition 2.2 Consider the CGTN $\circledast$, a linear space $W$, and MDF-valued $\Omega: W \rightarrow O^{+}$. In this case, we define a matrix Menger normed space (MMN-space) $(W, \Omega, \circledast)$ as follows:

(MMN1) $\Omega_{\tau}^{w}=\nabla_{\tau}^{0}$ for all $\tau>0$ if and only if $w=0$;

(MMN2) $\Omega_{\tau}^{\alpha w}=\Omega_{\frac{\tau}{|\alpha|}}^{w}$ for all $w \in W$ and $\alpha \in \mathbb{C}$ with $\alpha \neq 0$;

(MMN3) $\Omega_{\tau+\varsigma}^{w+w^{\prime}} \succeq \Omega_{\tau}^{w} \circledast \Omega_{\varsigma}^{w^{\prime}}$ for all $w, w^{\prime} \in W$ and $\tau, \varsigma \geq 0$.

A complete MMN-space is called MMB-space.

For example, the MDF-valued $\Omega$ given by

$$
\Omega_{\tau}^{w}= \begin{cases}0, & \text { if } \tau \leq 0, \\ \operatorname{diag}\left[\exp \left(-\frac{\|w\|}{\tau}\right), \frac{\tau}{\tau+\|w\|}, \exp \left(-\frac{\|w\|}{\tau}\right)\right], & \text { if } \tau>0,\end{cases}
$$

is a matrix Menger norm and $\left(W, \Omega, \circledast_{M}\right)$ is an MMN-space; here $(W,\|\cdot\|)$ is a normed linear space. 
Approximation of functional equations was studied in $\mathrm{MN}$-spaces, fuzzy metric spaces, and random multi-normed space $[16,17]$. Also stability results for stochastic fractional differential and integral equations were considered in [18-27].

Theorem $2.3([28,29])$ Let $(U, \rho)$ be a complete $\Xi_{4}$-valued metric space and let $\Lambda: U \rightarrow$ $U$ be a strictly contractive function with Lipschitz constant $\iota<1$. Then, for a given element $\xi \in U$, either

$$
\rho\left(\Lambda^{n} \xi, \Lambda^{n+1} \xi\right)=\infty
$$

for each $n \in \mathbb{N}$ or there is $n_{0} \in \mathbb{N}$ such that

(i) $\rho\left(\Lambda^{n} \xi, \Lambda^{n+1} \xi\right)<\infty$, for every $n \geq n_{0}$;

(ii) the fixed point $\zeta^{*}$ of $\Lambda$ is the limit point of the sequence $\left\{\Lambda^{n} \xi\right\}$;

(iii) in the set $V=\left\{\zeta \in U \mid \rho\left(\Lambda^{n_{0}} \xi, \zeta\right)<\infty\right\}, \zeta^{*}$ is the unique fixed point of $\Lambda$;

(iv) $(1-\iota) \rho\left(\zeta, \zeta^{*}\right) \leq \rho(\zeta, \Lambda \zeta)$ for every $\zeta \in V$.

Definition 2.4 ([30]) The Gamma function $\Gamma$ is defined by

$$
\Gamma(z)=\int_{0}^{\infty} e^{-\varsigma} \varsigma^{z-1} d \varsigma, \quad z \in \mathbb{C}, \operatorname{Re}(z)>0 .
$$

Let $\iota \in \Xi_{5}^{\circ}$, let $\Delta$ be an integrable function on $\Xi_{1}$ and $\Psi \in C^{1}\left(\Xi_{1}\right)$ an increasing function with $\Psi^{\prime}(\varsigma) \neq 0$, for each $\varsigma \in \Xi_{1}$. The $\Psi$-Hilfer fractional derivative is defined by [15]

$$
{ }^{H} \mathbb{D}_{0+}^{l, \kappa ; \Psi} \Delta(\varsigma)=\mathcal{I}_{0+}^{\kappa(1-\iota) ; \Psi}\left(\frac{1}{\Psi^{\prime}(\varsigma)} \frac{d}{d \varsigma}\right) \mathcal{I}_{0+}^{(1-\kappa)(1-\iota) ; \Psi} \Delta(\varsigma)
$$

Definition 2.5 If for every continuously differentiable function $\Delta(\varsigma)$ and MDF-valued $\varphi$ satisfying

$$
\Omega_{\tau}^{\left(H \mathbb{D}_{0+}^{\iota, \kappa ; \Psi} \Delta(\varsigma)-\boldsymbol{F}(\varsigma, \Delta(\varsigma))-\int_{0}^{\zeta} \boldsymbol{H}(\varsigma, \vartheta, \Delta(\varsigma)) d \vartheta\right)} \succeq \varphi_{\tau}^{\varsigma},
$$

for every $\varsigma \in \Xi_{1}$ and $\tau \in \Xi_{2}$, there exist a solution $\Delta_{0}(\varsigma)$ of the VIDE Eq. (1.1) and a fixed number $\lambda>0$ with

$$
\Omega_{\tau}^{\left(\Delta(\varsigma)-\Delta_{0}(\varsigma)\right)} \succeq \varphi_{\frac{\tau}{\lambda}}^{\varsigma}
$$

for every $\varsigma \in \Xi_{1}$ and $\tau \in \Xi_{2}$, where $\lambda$ is independent of $\Delta(\varsigma)$ and $\Delta_{0}(\varsigma)$, then (1.1) has the HUR stability.

\section{Main results}

Consider the following hypotheses:

(H0) Assume that $M, L_{\boldsymbol{F}}, L_{\boldsymbol{H}}$ are positive real numbers with $2 M\left(\max \left\{L_{\boldsymbol{F}}, L_{\boldsymbol{H}}\right\}\right) \in \Xi_{5}^{\circ}$ and let $\boldsymbol{F}: \Xi_{1} \times \mathbb{R} \rightarrow \mathbb{R}$ and $\boldsymbol{H}: \Xi_{1} \times \Xi_{1} \times \mathbb{R} \rightarrow \mathbb{R}$ be CFs satisfying

$$
\Omega_{\tau}^{\left(\boldsymbol{F}\left(\varsigma, \Delta_{1}\right)-\boldsymbol{F}\left(\varsigma, \Delta_{2}\right)\right)} \succeq \Omega_{\frac{\tau}{L_{\boldsymbol{F}}}}^{\Delta_{1}-\Delta_{2}}
$$


for all $\varsigma \in \Xi_{1}, \Delta_{1}, \Delta_{2} \in \mathbb{R}$ and $\tau \in \Xi_{2}$, and

$$
\Omega_{\tau}^{\left(\boldsymbol{H}\left(\varsigma, \vartheta, \Delta_{1}\right)-\boldsymbol{H}\left(\varsigma, \vartheta, \Delta_{2}\right)\right)} \succeq \Omega_{\frac{\tau}{L_{\boldsymbol{H}}}}^{\Delta_{1}-\Delta_{2}},
$$

for all $\varsigma, \vartheta \in \Xi_{1}, \Delta_{1}, \Delta_{2} \in \mathbb{R}$ and $\tau \in \Xi_{2}$.

Theorem 3.1 Suppose $(H 0)$ holds and consider a nondecreasing function $\Psi \in C\left(\Xi_{1}\right)$ with $\Psi^{\prime}(\varsigma) \neq 0$ and $\operatorname{CDF} \Delta: \Xi_{1} \rightarrow \mathbb{R}$ satisfying

$$
\Omega_{\tau}^{\left(H_{\mathbb{D}_{0+}^{l, \kappa ; \Psi}} \Delta(\varsigma)-\boldsymbol{F}(\varsigma, \Delta(\varsigma))-\int_{0}^{\zeta} \boldsymbol{H}(\varsigma, \vartheta, \Delta(\vartheta)) d \vartheta\right)} \succeq \varphi_{\tau}^{\zeta}
$$

for all $\varsigma, \vartheta \in \Xi_{1}, \Delta \in \mathbb{R}$, and $\tau \in \Xi_{2}$, where $\varphi$ is $M D F$-valued with

$$
\mathcal{I}_{0+}^{l ; \Psi} \Delta(\varsigma):=\frac{1}{\Gamma(\iota)} \int_{0}^{\varsigma} \Psi^{\prime}(\xi)(\Psi(\varsigma)-\Psi(\xi))^{\iota-1} \Delta(\xi) d \xi
$$

satisfying

$$
\Omega_{\tau}^{\Delta(\varsigma)} \succeq \varphi_{\tau}^{\varsigma} \quad \Longrightarrow \quad \Omega_{\tau}^{\mathcal{I}_{0_{+}}^{l ; \Psi} \Delta(\varsigma)} \succeq \varphi_{\frac{\tau}{M}}^{\varsigma}, \quad \inf _{\xi \in \Xi_{1}} \varphi_{\frac{\tau}{T}}^{\xi} \succeq \varphi_{\tau}^{\varsigma},
$$

for each $\varsigma \in \Xi_{1}$ and $\tau \in \Xi_{2}$. Then, we can find a unique $C F \Delta_{0}: \Xi_{1} \rightarrow \mathbb{R}$ such that

$$
\begin{aligned}
\Delta_{0}(\varsigma)= & \frac{(\Psi(\varsigma)-\Psi(0))^{\gamma-1}}{\Gamma(\gamma)} \sigma \\
& +\mathcal{I}_{0+}^{l ; \Psi} \boldsymbol{F}\left(\varsigma, \Delta_{0}(\varsigma)\right) \\
& +\mathcal{I}_{0+}^{l ; \Psi}\left[\int_{0}^{\xi} \boldsymbol{H}\left(\varsigma, \vartheta, \Delta_{0}(\vartheta)\right) d \vartheta\right]
\end{aligned}
$$

with $\mathcal{I}_{0+}^{1-\gamma ; \Psi} \Delta(0)=\sigma, \iota \in \stackrel{\circ}{\Xi}_{5}, \kappa \in \Xi_{5}$, and

$$
\Omega_{\tau}^{\left(\Delta(\varsigma)-\Delta_{0}(\varsigma)\right)} \succeq \varphi_{\frac{M \tau}{1-2 M\left(\max \left\{L_{\boldsymbol{F}}, L_{\boldsymbol{H}}\right\}\right)}}^{\zeta}
$$

for each $\varsigma \in \Xi_{1}$ and $\tau \in \Xi_{2}$.

Proof For $\alpha, \beta \in U$, we set

$$
\rho(\alpha, \beta)=\inf \left\{\lambda \in \Xi_{4}: \Omega_{\tau}^{(\alpha(\varsigma)-\beta(\varsigma))} \succeq \varphi_{\frac{\tau}{\lambda}}^{\varsigma}\right\},
$$

for each $\varsigma \in \Xi_{1}$ and $\tau \in \Xi_{2}$, where

$$
U=\left\{\alpha: \Xi_{1} \rightarrow \mathbb{R} \text { is a } \mathrm{CF}\right\}
$$

Let $\Lambda: U \rightarrow U$ be given by

$$
\Lambda \alpha(\varsigma)=\frac{(\Psi(\varsigma)-\Psi(0))^{\gamma-1}}{\Gamma(\gamma)} \sigma
$$




$$
\begin{aligned}
& +\mathcal{I}_{0+}^{i ; \Psi} \boldsymbol{F}(\varsigma, \alpha(\varsigma)) \\
& +\mathcal{I}_{0+}^{i ; \Psi}\left[\int_{0}^{\xi} \boldsymbol{H}(\varsigma, \vartheta, \alpha(\vartheta)) d \vartheta\right],
\end{aligned}
$$

for all $\alpha \in \Xi_{1}$ and $\varsigma \in \Xi_{1}$.

First we show that $\Lambda$ is strictly contractive on $U$. Let $\lambda_{\alpha \beta} \in \Xi_{4}$ be a fixed number with $\rho(\alpha, \beta) \leq \lambda_{\alpha \beta}$ for any $\alpha, \beta \in U$, so from Eq. (3.8) we have

$$
\Omega_{\tau}^{(\alpha(\varsigma)-\beta(\varsigma))} \succeq \varphi_{\frac{\tau}{\lambda_{\alpha \beta}}}^{\varsigma}
$$

Let $0=\varpi_{1}<\varpi_{2}<\cdots<\varpi_{k}=T, \Delta \xi_{i}=\varpi_{i}-\varpi_{i-1}=\frac{|T-0|}{k}, i=1,2, \ldots, k$ and $\|\Delta \xi\|=$ $\max _{1 \leq i \leq k}\left(\Delta \xi_{i}\right)$, for each $\varsigma, \xi \in \Xi_{1}$ and $\tau \in \Xi_{2}$. From Eqs. (3.2), (3.5), and (3.10), we have

$$
\begin{aligned}
& \Omega_{\tau}^{\left(\int_{0}^{\xi} \boldsymbol{H}(\varsigma, \vartheta, \alpha(\vartheta))-\boldsymbol{H}(\varsigma, \vartheta, \beta(\vartheta)) d \vartheta\right)} \\
& =\Omega_{\tau}^{\left(\lim _{\|\Delta \xi\| \rightarrow 0} \sum_{i=1}^{k} \boldsymbol{H}\left(\zeta, \varpi_{i}, \alpha\left(\varpi_{i}\right)\right)-\boldsymbol{H}\left(\zeta, \varpi_{i}, \beta\left(\varpi_{i}\right)\right) \Delta \xi_{i}\right)} \\
& =\lim _{\|\Delta \xi\| \rightarrow 0} \Omega_{\tau}^{\left(\sum_{i=1}^{k}\left(\boldsymbol{H}\left(\varsigma, \varpi_{i}, \alpha\left(\varpi_{i}\right)\right)-\boldsymbol{H}\left(\varsigma, \varpi_{i}, \beta\left(\varpi_{i}\right)\right) \Delta \xi_{i}\right)\right)} \\
& \lim _{\|\Delta \xi\| \rightarrow 0} \circledast_{M} \Omega_{\frac{\tau}{k}}^{\left(\boldsymbol{H}\left(\varsigma, \varpi_{i}, \alpha\left(\varpi_{i}\right)\right)-\boldsymbol{H}\left(\varsigma, \varpi_{i}, \beta\left(\varpi_{i}\right)\right) \Delta \xi_{i}\right)} \\
& \succeq \inf _{\xi \in \Xi_{1}} \Omega_{\frac{\tau}{(\boldsymbol{H}(s, \xi, \alpha(\xi))-\boldsymbol{H}(s, \xi, \beta(\xi)))}}^{\left(k \Delta \xi_{i}\right.} \\
& \inf _{\xi \in \Xi_{1}} \Omega_{\tau}^{(\boldsymbol{H}(\zeta, \xi, \alpha(\xi))-\boldsymbol{H}(\varsigma, \xi, \beta(\xi)))} \\
& \succeq \inf _{\xi \in \Xi_{1}} \varphi_{\frac{\xi}{T \lambda \alpha \beta} L_{H}}^{\tau} \\
& \succeq \varphi_{\frac{\tau}{\lambda_{\alpha \beta} L_{H}}},
\end{aligned}
$$

Then, by Eqs. (3.1), (3.4), (3.5), (3.9), (3.10), and (3.11), we have

$$
\begin{aligned}
& \Omega_{\tau}^{(\Lambda \alpha(\varsigma)-\Lambda \beta(\varsigma))} \\
& =\Omega_{\tau}^{\left(\frac{1}{\Gamma(l)} \int_{0}^{\zeta} \Psi^{\prime}(\xi)(\Psi(\varsigma)-\Psi(\xi))^{l-1}\left(\boldsymbol{F}(\xi, \alpha(\xi))-\boldsymbol{F}(\xi, \beta(\xi))+\int_{0}^{\xi} \boldsymbol{H}(\varsigma, \vartheta, \alpha(\vartheta))-\boldsymbol{H}(\varsigma, \vartheta, \beta(\vartheta)) d \vartheta\right) d \xi\right)} \\
& \succeq \Omega_{\frac{\tau}{2}}^{\left(\mathcal{I}_{0+}^{l, \Psi}(\boldsymbol{F}(\xi, \alpha(\xi))-\boldsymbol{F}(\xi, \beta(\xi))) d \xi\right)} \circledast_{M} \Omega_{\frac{\tau}{2}}^{\left(\mathcal{I}_{0+}^{l i}\left(\int_{0}^{\xi} \boldsymbol{H}(\varsigma, \vartheta, \alpha(\vartheta))-\boldsymbol{H}(\varsigma, \vartheta, \beta(\vartheta)) d \vartheta\right)\right)} \\
& \succeq \varphi_{\frac{\tau}{2 M \lambda_{\alpha \beta} L_{\boldsymbol{F}}}} \circledast_{M} \varphi_{\frac{\tau}{2 M \lambda_{\alpha \beta} L_{\boldsymbol{H}}}}^{\varsigma} \\
& \succeq \varphi_{\frac{\tau}{2 M \lambda_{\alpha \beta}\left(\max \left\{L_{\boldsymbol{F}}, L_{\boldsymbol{H}}\right\}\right)}},
\end{aligned}
$$

and we conclude that

$$
\rho(\Lambda \alpha, \Lambda \beta) \leq 2 M \lambda_{\alpha \beta}\left(\max \left\{L_{\boldsymbol{F}}, L_{\boldsymbol{H}}\right\}\right)
$$

for all $\varsigma \in \Xi_{1}$ and $\tau \in \Xi_{2}$. Hence, we deduce that $\rho(\Lambda \alpha, \Lambda \beta) \leq\left[2 M\left(\max \left\{L_{\boldsymbol{F}}, L_{\boldsymbol{H}}\right\}\right)\right] \rho(\alpha, \beta)$ for any $\alpha, \beta \in U$, where $2 M\left(\max \left\{L_{\boldsymbol{F}}, L_{\boldsymbol{H}}\right\}\right) \in \Xi_{5}^{\circ}$. 
From Eq. (3.9), we can find a fixed number $\lambda \in \Xi_{2}$ such that

$$
\begin{aligned}
& \Omega_{\tau}^{\left(\Lambda \beta(\varsigma)-\beta_{0}(\varsigma)\right)} \\
& \quad=\Omega_{\tau}^{\left(\frac{(\Psi(\varsigma)-\Psi(0))^{\gamma-1}}{\Gamma(\gamma)} \sigma+\mathcal{I}_{0+}^{l ;} \boldsymbol{F}\left(\varsigma, \beta_{0}(\varsigma)\right)+\mathcal{I}_{0+}^{l ;}\left[\int_{0}^{\xi} \boldsymbol{H}\left(\varsigma, \vartheta, \beta_{0}(\vartheta)\right) d \vartheta\right]-\beta_{0}(\varsigma)\right)} \\
& \quad \succeq \varphi_{\frac{\tau}{\lambda}}^{\varsigma}
\end{aligned}
$$

for arbitrary $\beta_{0} \in U$, for all $\varsigma \in \Xi_{1}$ and $\tau \in \Xi_{2}$. The boundedness property of

$$
\boldsymbol{F}\left(\varsigma, \beta_{0}(\varsigma)\right), \quad \boldsymbol{H}\left(\varsigma, \vartheta, \beta_{0}(\vartheta)\right), \quad \beta_{0}(\varsigma)
$$

$\min _{\varsigma \in \Xi_{1}} \varphi_{\tau}^{\varsigma}>0$, and Eq. (3.8) imply that $\rho\left(\Lambda \beta, \beta_{0}\right)<\infty$. From Theorem 2.3 , there exists a CF $\Delta_{0}: \Xi_{1} \rightarrow \mathbb{R}$ such that $\Lambda^{n} \Delta_{0} \rightarrow \Delta_{0}$ in $(U, \rho)$ and $\Lambda \Delta_{0}=\Delta_{0}$.

Since $\beta$ and $\Delta_{0}$ are bounded on $\Xi_{1}$ for each $\beta \in U$ and $\min _{\varsigma \in \Xi_{1}} \varphi_{\tau}^{\varsigma}>0$, we have a fixed number $\lambda_{\beta} \in \Xi_{4}$ with

$$
\Omega_{\tau}^{\left(\beta_{0}(\varsigma)-\beta(\varsigma)\right)} \succeq \varphi_{\frac{\tau}{\lambda_{\beta}}}^{\varsigma}
$$

for any $\varsigma \in \Xi_{1}$ and $\tau \in \Xi_{2}$. Thus $\rho\left(\beta_{0}, \beta\right)<\infty$ for any $\beta \in U$.

Therefore, $U=\left\{\beta \in U: \rho\left(\beta_{0}, \beta\right)<\infty\right\}$. Also Theorem 2.3 and Eq. (3.6) imply the uniqueness of $\Delta_{0}$.

Using Eqs. (3.3), (3.5), and (3.9), we have

$$
\Omega_{\tau}^{\left(\Delta(\varsigma)-\frac{(\Psi(\varsigma)-\Psi(0))^{\gamma-1}}{\Gamma(\gamma)} \sigma-\mathcal{I}_{0+}^{l ; \Psi} \boldsymbol{F}(\varsigma, \Delta(\varsigma))-\mathcal{I}_{0+}^{l ; \Psi}\left[\int_{0}^{\xi} \boldsymbol{H}(\varsigma, \vartheta, \Delta(\vartheta)) d \vartheta\right]\right)} \geq \varphi_{\frac{\tau}{M}}^{\varsigma} .
$$

Then, we obtain

$$
\Omega_{\tau}^{(\Delta(\varsigma)-\Lambda \Delta(\varsigma))} \succeq \varphi_{\frac{\tau}{M}}^{\varsigma}
$$

for any $\varsigma \in \Xi_{1}$ and $\tau \in \Xi_{2}$, which implies

$$
\rho(\Delta, \Lambda \Delta) \leq M
$$

From Theorem 2.3 and Eq. (3.13), we deduce that

$$
\rho\left(\Delta, \Delta_{0}\right) \leq \frac{1}{1-2 M\left(\max \left\{L_{\boldsymbol{F}}, L_{\boldsymbol{H}}\right\}\right)} \rho(\Lambda \Delta, \Delta) \leq \frac{M}{1-2 M\left(\max \left\{L_{\boldsymbol{F}}, L_{\boldsymbol{H}}\right\}\right)},
$$

which implies Eq. (3.7).

Theorem 3.2 Assume that $\iota, \kappa \in \Xi_{5}^{\circ}$ and consider a nondecreasing function $\Psi \in C^{1}\left(\Xi_{1}\right)$ with $\Psi^{\prime}(\varsigma) \neq 0$ for all $\varsigma \in \Xi_{1}$. Also let $L_{\boldsymbol{F}}, L_{\boldsymbol{H}} \in \Xi_{2}$ be fixed numbers such that $2 M\left(\max \left\{L_{\boldsymbol{F}}\right.\right.$, $\left.\left.T L_{\boldsymbol{H}}\right\}\right) \in \Xi_{5}^{\circ}$. Consider CFs $\boldsymbol{F}: \boldsymbol{\Xi}_{1} \times \mathbb{R} \rightarrow \mathbb{R}$ and $\boldsymbol{H}: \boldsymbol{\Xi}_{1} \times \Xi_{1} \times \mathbb{R} \rightarrow \mathbb{R}$ satisfying Eqs. (3.1) and (3.2), respectively. If for $\varepsilon \geq 0, \tau \in \Xi_{2}, \boldsymbol{\epsilon}_{\tau}:=\operatorname{diag}\left[e^{-\frac{\varepsilon}{\tau}}, \ldots, e^{-\frac{\varepsilon}{\tau}}\right]$, a CDF $\Delta: \Xi_{1} \rightarrow \mathbb{R}$ satisfies

$$
\Omega_{\tau}^{\left(H_{\mathbb{D}^{\ell+}}^{\ell, \kappa ; \psi} \Delta(\varsigma)-\boldsymbol{F}(\varsigma, \Delta(\varsigma))-\int_{0}^{\zeta} \boldsymbol{H}(\varsigma, \vartheta, \Delta(\vartheta)) d \vartheta\right)} \succeq \boldsymbol{\epsilon}_{\tau}
$$


for all $\varsigma, \vartheta \in \Xi_{1}, \Delta \in \mathbb{R}$, and $\tau \in \Xi_{2}$, then we can find a unique CF $\Delta_{0}: \Xi_{1} \rightarrow \mathbb{R}$ satisfying Eq. (3.6) and

$$
\Omega_{\tau}^{\left(\Delta(\varsigma)-\Delta_{0}(\varsigma)\right)} \succeq \boldsymbol{\epsilon} \frac{M \tau}{1-2 M\left(\max \left\{L_{\boldsymbol{F}}, T L_{\boldsymbol{H}}\right\}\right)},
$$

for all $\varsigma \in \Xi_{1}$ and $\Delta \in \mathbb{R}$.

Proof Let $U=\left\{\alpha: \Xi_{1} \rightarrow \mathbb{R}\right.$ is a CF $\}$. Consider the $\Xi_{4}$-valued metric on $U$ defined by

$$
\rho(\alpha, \beta)=\inf \left\{\lambda \in \Xi_{4}: \Omega_{\tau}^{(\alpha(\varsigma)-\beta(\varsigma))} \succeq \epsilon_{\frac{\tau}{\lambda}}\right\}
$$

for each $\varsigma \in \Xi_{1}$ and $\tau \in \Xi_{2}$. In [15] the authors proved the completeness of $(U, \rho)$.

Let $\Lambda: U \rightarrow U$ be given by

$$
\begin{aligned}
\Lambda \alpha(\varsigma)= & \frac{(\Psi(\varsigma)-\Psi(0))^{\gamma-1}}{\Gamma(\gamma)} \sigma \\
& +\mathcal{I}_{0+}^{\prime ; \Psi} \boldsymbol{F}(\varsigma, \alpha(\varsigma)) \\
& +\mathcal{I}_{0+}^{\iota ; \Psi}\left[\int_{0}^{\xi} \boldsymbol{H}(\varsigma, \vartheta, \alpha(\vartheta)) d \vartheta\right]
\end{aligned}
$$

for all $\varsigma \in \Xi_{1}$.

Let $\alpha, \beta \in U$ and consider a fixed number $\lambda_{\alpha \beta} \in \Xi_{4}$ such that $\rho(\alpha, \beta) \leq \lambda_{\alpha \beta}$ and

$$
\Omega_{\tau}^{(\alpha(\varsigma)-\beta(\varsigma))} \succeq \epsilon_{\frac{\tau}{\lambda_{\alpha \beta}}}
$$

Let $0=\varpi_{1}<\varpi_{2}<\cdots<\varpi_{k}=T, \Delta \xi_{i}=\varpi_{i}-\varpi_{i-1}=\frac{|T-0|}{k}, i=1,2, \ldots, k$, and $\|\Delta \xi\|=$ $\max _{1 \leq i \leq k}\left(\Delta \xi_{i}\right)$, for each $\varsigma \in \Xi_{1}$ and $\tau \in \Xi_{2}$.

From Eqs. (3.2) and (3.17), we have

$$
\begin{aligned}
& \Omega_{\tau}^{\left(\int_{0}^{\xi} \boldsymbol{H}(\varsigma, \vartheta, \alpha(\vartheta))-\boldsymbol{H}(\varsigma, \vartheta, \beta(\vartheta)) d \vartheta\right)} \\
& =\Omega_{\tau}^{\left(\lim _{\|\Delta \xi\| \rightarrow 0} \sum_{i=1}^{k} \boldsymbol{H}\left(\varsigma, \varpi_{i}, \alpha\left(\varpi_{i}\right)\right)-\boldsymbol{H}\left(\varsigma, \varpi_{i}, \beta\left(\varpi_{i}\right)\right) \Delta \xi_{i}\right)} \\
& =\lim _{\|\Delta \xi\| \rightarrow 0} \Omega_{\tau}^{\left(\sum_{i=1}^{k}\left(\boldsymbol{H}\left(\varsigma, \varpi_{i}, \alpha\left(\varpi_{i}\right)\right)-\boldsymbol{H}\left(\varsigma, \varpi_{i}, \beta\left(\varpi_{i}\right)\right) \Delta \xi_{i}\right)\right)}
\end{aligned}
$$

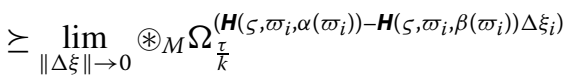

$$
\begin{aligned}
& \succeq \inf _{\xi \in \Xi_{1}} \Omega_{\frac{\tau}{k \Delta \xi_{i}}}^{(\boldsymbol{H}(\varsigma, \xi, \alpha(\xi))-\boldsymbol{H}(\varsigma, \xi, \beta(\xi)))} \\
& \succeq \inf _{\xi \in \Xi_{1}} \Omega_{\frac{\tau}{T}}^{(\boldsymbol{H}(\varsigma, \xi, \alpha(\xi))-\boldsymbol{H}(\varsigma, \xi, \beta(\xi)))} \\
& \succeq \inf _{\xi \in \Xi_{1}} \boldsymbol{\epsilon}_{\frac{\tau}{T \lambda_{\alpha \beta} L_{H}}} \\
& =\epsilon_{\frac{\tau}{T \lambda_{\alpha \beta} L_{H}}},
\end{aligned}
$$


Then, by Eqs. (3.1), (3.16), and (3.17), we have

$$
\begin{aligned}
& \Omega_{\tau}^{(\Lambda \alpha(\varsigma)-\Lambda \beta(\varsigma))} \\
& \quad=\Omega_{\tau}^{\left(\frac{1}{\Gamma(l)} \int_{0}^{\zeta} \Psi^{\prime}(\xi)(\Psi(\varsigma)-\Psi(\xi))^{\ell-1}\left(\boldsymbol{F}(\xi, \alpha(\xi))-\boldsymbol{F}(\xi, \beta(\xi))+\int_{0}^{\xi} \boldsymbol{H}(\varsigma, \vartheta, \alpha(\vartheta))-\boldsymbol{H}(\varsigma, \vartheta, \beta(\vartheta)) d \vartheta\right) d \xi\right)} \\
& \quad \succeq \Omega_{\frac{\tau}{2}}^{\left(\mathcal{I}_{0+}^{l ;}(\boldsymbol{F}(\xi, \alpha(\xi))-\boldsymbol{F}(\xi, \beta(\xi))) d \xi\right)} \circledast_{M} \Omega_{\frac{\tau}{2}}^{\left(\mathcal{I}_{0+}^{l ;}\left(\int_{0}^{\xi} \boldsymbol{H}(\varsigma, \vartheta, \alpha(\vartheta))-\boldsymbol{H}(\varsigma, \vartheta, \beta(\vartheta)) d \vartheta\right)\right)} \\
& \quad \succeq \boldsymbol{\epsilon}_{\frac{\tau}{2 M \lambda_{\alpha \beta} L_{\boldsymbol{F}}}} \circledast_{M} \boldsymbol{\epsilon}_{\frac{\tau}{2 M T \lambda_{\alpha \beta} L_{\boldsymbol{H}}}} \\
& \quad \succeq \boldsymbol{\epsilon}_{\frac{\tau}{2 M \lambda_{\alpha \beta}\left(\max \left\{L_{\boldsymbol{F}}, T L_{\boldsymbol{H}}\right\}\right)}},
\end{aligned}
$$

for each $\zeta \in \Xi_{1}$ and $\tau \in \Xi_{2}$. Therefore $\rho(\Lambda \alpha, \Lambda \beta) \leq\left[2 M\left(\max \left\{L_{\boldsymbol{F}}, T L_{\boldsymbol{H}}\right\}\right)\right] \rho(\alpha, \beta)$ for any $\alpha, \beta \in U$, where $2 M\left(\max \left\{L_{\boldsymbol{F}}, T L_{\boldsymbol{H}}\right\}\right) \in \Xi_{5}^{\circ}$.

From Eq. (3.16), we can find a fixed number $\lambda \in \Xi_{2}$ such that

$$
\begin{aligned}
& \Omega_{\tau}^{\left(\Lambda \beta(\varsigma)-\beta_{0}(\varsigma)\right)} \\
& \quad=\Omega_{\tau}^{\left(\frac{(\Psi(\varsigma)-\Psi(0))^{\gamma-1}}{\Gamma(\gamma)} \sigma+\mathcal{I}_{0+}^{l ; \Psi} \boldsymbol{F}\left(\varsigma, \beta_{0}(\varsigma)\right)+\mathcal{I}_{0+}^{l ; \Psi}\left[\int_{0}^{\xi} \boldsymbol{H}\left(\varsigma, \vartheta, \beta_{0}(\vartheta)\right) d \vartheta\right]-\beta_{0}(\varsigma)\right)} \\
& \quad \succeq \boldsymbol{\epsilon} \frac{\tau}{\lambda},
\end{aligned}
$$

for arbitrary $\beta_{0} \in U$, for all $\varsigma \in \Xi_{1}$ and $\tau \in \Xi_{2}$. The boundedness property of

$$
\boldsymbol{F}\left(\varsigma, \beta_{0}(\varsigma)\right), \boldsymbol{H}\left(\varsigma, \vartheta, \beta_{0}(\vartheta)\right), \beta_{0}(\varsigma)
$$

and Eq. (3.15) imply that $\rho\left(\Lambda \beta, \beta_{0}\right)<\infty$. From Theorem 2.3 , there exists a CF $\Delta_{0}: \Xi_{1} \rightarrow \mathbb{R}$ such that $\Lambda^{n} \Delta_{0} \rightarrow \Delta_{0}$ in $(U, \rho)$ and $\Lambda \Delta_{0}=\Delta_{0}$. Using a method similar to that in the proof of Theorem 3.1, we get $\left\{\beta \in U: \rho\left(\beta_{0}, \beta\right)<\infty\right\}=U$. Also Theorem 2.3 and Eq. (3.6) imply the uniqueness of $\Delta_{0}$.

Now, using Eq. (3.3) and [12, Theorem 5], we have

$$
\Omega_{\tau}^{\left(\Delta(\varsigma)-\frac{(\Psi(\varsigma)-\Psi(0))^{\gamma-1}}{\Gamma(\gamma)} \sigma-\mathcal{I}_{0+}^{i ; \Psi} \boldsymbol{F}\left(\varsigma, \Delta_{0}(\varsigma)\right)-\mathcal{I}_{0+}^{i ;}\left[\int_{0}^{\xi} \boldsymbol{H}\left(\varsigma, \vartheta, \Delta_{0}(\vartheta)\right) d \vartheta\right]\right)} \succeq \boldsymbol{\epsilon}_{\frac{\tau}{M}},
$$

for all $\varsigma \in \Xi_{1}$, which implies

$$
\rho(\Delta, \Lambda \Delta) \leq M
$$

From Theorem 2.3 and Eq. (3.8), we deduce that

$$
\Omega_{\tau}^{\left(\Delta(\varsigma)-\Delta_{0}(\varsigma)\right)} \succeq \boldsymbol{\epsilon} \frac{M \tau}{1-2 M\left(\max \left\{L_{\boldsymbol{F}}, T L_{\boldsymbol{H}}\right\}\right)},
$$

which implies Eq. (3.14) for all $\varsigma \in \Xi_{1}$.

\section{Conclusions}

We introduced a new model of stochastic matrix control functions which helped us to stabilize a pseudo-nonlinear fractional Volterra integral equation and get better approximation for it. In fact, two kinds of novel stability concepts, i.e., Hyers-Ulam-Rassias and 
Hyers-Ulam stability, of a fractional Volterra integral equation with delay are proved by using an alternative fixed point theorem in generalized complete metric spaces and the concept of stochastic matrix control functions in a matrix MB-space.

\section{Acknowledgements}

The authors are thankful to the area editor and anonymous referees for giving valuable comments and suggestions.

\section{Funding}

No funding.

Availability of data and materials

Not applicable.

\section{Competing interests}

The authors declare that they have no competing interests.

\section{Authors' contributions}

All authors conceived of the study, participated in its design and coordination, drafted the manuscript, participated in the sequence alignment, and read and approved the final manuscript.

\section{Publisher's Note}

Springer Nature remains neutral with regard to jurisdictional claims in published maps and institutional affiliations.

Received: 1 October 2020 Accepted: 3 February 2021 Published online: 22 February 2021

\section{References}

1. Kilbas, A.A., Srivastava, H.M., Trujillo, J.J.: Theory and Applications of Fractional Differential Equations. Elsevier, Amsterdam (2006) 204

2. Sousa, J.V.d.C., de Oliveira, E.C., Magna, L.A.: Fractional calculus and the ESR test. AIMS Math. 2(4), $692-705$ (2017)

3. Wang, J.R., Feckan, M., Zhou, Y.: A survey on impulsive fractional differential equations. Fract. Calc. Appl. Anal. 19(4), 806-831 (2016)

4. Liang, X., Gao, F., Zhou, C.-B., Wang, Z., Yang, X.-J.: An anomalous diffusion model based on a new general fractional operator with the Mittag-Leffler function of Wiman type. Adv. Differ. Equ. 2018, Paper No. 25, 11 pp. (2018)

5. Gumah, G., Al-Omari, S., Baleanu, D.: Soft computing technique for a system of fuzzy Volterra integro-differential equations in a Hilbert space. Appl. Numer. Math. 152, 310-322 (2020)

6. Sun, H.-G., Sheng, H., Chen, Y.-Q., Chen, W., Yu, Z.-B. A dynamic-order fractional dynamic system. Chin. Phys. Lett. 30(4), $046601(2013)$

7. Sun, H., Chen, W., Li, C., Chen, Y.: Finite difference schemes for variable-order time fractional diffusion equation. Int. J. Bifurc. Chaos, 22(04), 1250085 (2012)

8. Wang, J.R., Zhou, Y.: Mittag-Leffler-Ulam stabilities of fractional evolution equations. Appl. Math. Lett. 25(4), 723-728 (2012)

9. Sousa, J.V.d.C., Kucche, K.D., de Oliveira, E.C.: On the Ulam-Hyers stabilities of the solutions of $\Psi$-Hilfer fractional differential equation with abstract Volterra operator. Math. Methods Appl. Sci. 42(9), 3021-3032 (2019)

10. Muniyappan, P., Rajan, S.: Stability of a class of fractional integro-differential equation with nonlocal initial conditions. Acta Math. Univ. Comen. 87(1), 85-95 (2018)

11. Selvam, A.G.M., Baleanu, D., Alzabut, J., Vignesh, D., Abbas, S.: On Hyers-Ulam Mittag-Leffler stability of discrete fractional Duffing equation with application on inverted pendulum. Adv. Differ. Equ. 2020, 456 (2020)

12. Sousa, J.V.d.C., de Oliveira, E.C.: On the $\Psi$-Hilfer fractional derivative. Commun. Nonlinear Sci. Numer. Simul. 60, 72-91 (2018)

13. Schweizer, B., Sklar, A.: Probabilistic Metric Spaces. North-Holland Series in Probability and Applied Mathematics. North-Holland, New York (1983)

14. Hadžić, O., Pap, E.: Fixed Point Theory in Probabilistic Metric Spaces. Mathematics and Its Applications, vol. 536. Kluwer Academic, Dordrecht (2001)

15. Saadati, R.: Random Operator Theory. Elsevier, London (2016)

16. El-Moneam, M.A., Ibrahim, T.F., Elamody, S.: Stability of a fractional difference equation of high order. J. Nonlinear Sci. Appl. 12(2), 65-74 (2019)

17. Madadi, M., Saadati, R., De la Sen, M.: Stability of unbounded differential equations in Menger k-normed spaces: a fixed point technique. Mathematics 8(3), 400 (2020)

18. Constantinescu, C.D., Ramirez, J.M., Zhu, W.R.: An application of fractional differential equations to risk theory. Finance Stoch. 23(4), 1001-1024 (2019)

19. El-Sayed, A.M.A., Gaafar, F.M.: Existence and uniqueness of solution for Sturm-Liouville fractional differential equation with multi-point boundary condition via Caputo derivative. Adv. Differ. Equ. 2019, Paper No. 46, 17 pp. (2019)

20. Jiang, J. O'Regan, D, Xu, J, Fu, Z: Positive solutions for a system of nonlinear Hadamard fractional differential equations involving coupled integral boundary conditions. J. Inequal. Appl. 2019, Paper No. 204, 18 pp. (2019)

21. Sene, N.: Stability analysis of the generalized fractional differential equations with and without exogenous inputs. J. Nonlinear Sci. Appl. 12(9), 562-572 (2019)

22. Sene, N.: Global asymptotic stability of the fractional differential equations. J. Nonlinear Sci. Appl. 13(3), 171-175 (2020) 
23. Ali, A., Shah, K., Li, Y., Khan, R.A.: Numerical treatment of coupled system of fractional order partial differential equations. J. Math. Comput. Sci. 19, 74-85 (2019)

24. Pap, E., Park, C., Saadati, R.: Additive $\sigma$-random operator inequality and rhom-derivations in fuzzy Banach algebras. Sci. Bull. “Politeh." Univ. Buchar., Ser. A, Appl. Math. Phys. 82(2), 3-14 (2020)

25. Asaduzzaman, M., Kilicman, A., Ali, M.Z.: Presence and diversity of positive solutions for a Caputo-type fractional order nonlinear differential equation with an advanced argument. J. Math. Comput. Sci. 23, 230-244 (2021)

26. Chaharpashlou, R., Saadati, R., Atangana, A.: Ulam-Hyers-Rassias stability for nonlinear $\Psi$-Hilfer stochastic fractional differential equation with uncertainty. Adv. Differ. Equ. 2020, Paper No. 339, 10 pp. (2020)

27. Madadi, M., Saadati, R., Park, C., Rassias, J.M.: Stochastic Lie bracket (derivation, derivation) in MB-algebras. J. Inequal. Appl. 2020, Paper No. 141, 15 pp. (2020)

28. Cădariu, L., Radu, V.: Fixed points and the stability of Jensen's functional equation. JIPAM. J. Inequal. Pure Appl. Math. 4(1), Article ID 4 (2003)

29. Diaz, J.B., Margolis, B.: A fixed point theorem of the alternative, for contractions on a generalized complete metric space. Bull. Am. Math. Soc. 74, 305-309 (1968)

30. Sousa, J.V.d.C., de Oliveira, E.C.: On a new operator in fractional calculus and applications. J. Fixed Point Theory Appl. 20(3), Paper No. 96,21 pp. (2018)

\section{Submit your manuscript to a SpringerOpen ${ }^{\circ}$ journal and benefit from:}

- Convenient online submission

- Rigorous peer review

- Open access: articles freely available online

- High visibility within the field

- Retaining the copyright to your article

Submit your next manuscript at $\boldsymbol{~ s p r i n g e r o p e n . c o m ~}$ 